\title{
Parenchymal Hematoma as Hemorrhagic Transformation of Ischemic Stroke Secondary to Spontaneous Internal Carotid Artery Dissection
}

\author{
Eugenia Rota Paolo Immovilli Nicola Morelli Paola De Mitri \\ Fabiola Magnifico Emilio Terlizzi Donata Guidetti \\ Department of Neurology, G. da Saliceto Hospital, Piacenza, Italy
}

Dear Sir,

Hemorrhagic transformation has rarely been reported in the evolution of ischemic stroke caused by carotid artery dissection [1].

Herein, we report the neuroimaging of a patient admitted to the hospital for a parenchymal hematoma over the right basal ganglia which, after an extensive neuroimaging workup, turned out to be the hemorrhagic transformation of an ischemic stroke secondary to spontaneous internal carotid artery dissection. This case was not treated with r-TPA or endovascular therapy.
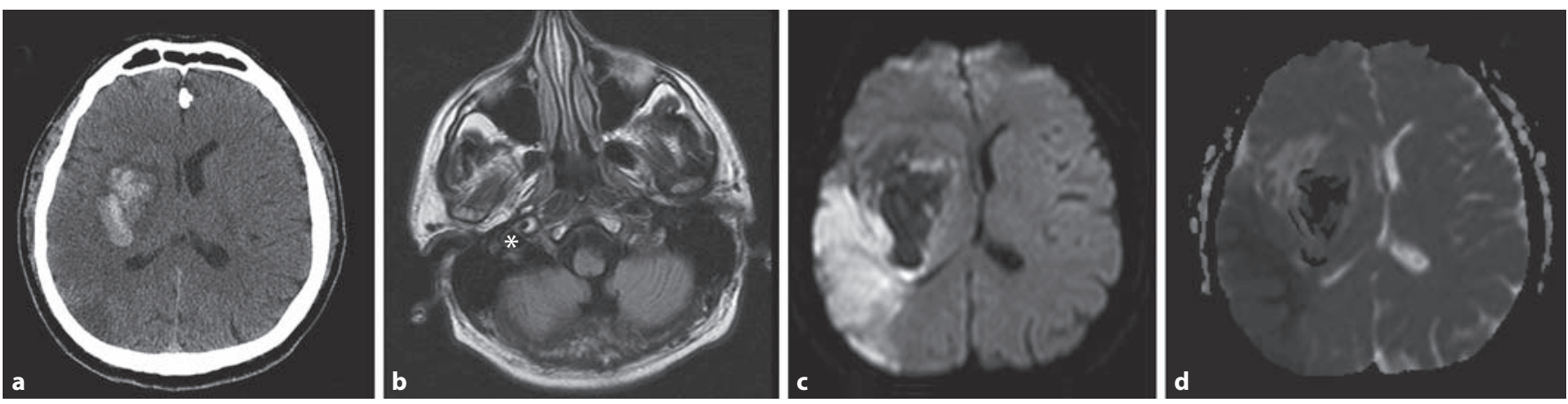

Fig. 1. CT brain scan of the brain ( $13 \mathrm{~h}$ after the onset of headache) showing the right intraparenchymal hematoma (a). $\mathrm{T}_{1}$-weighted cervical, axial MRI scans (2 days after the onset of left hemiplegia) showing a semilunar, crescent-shaped, hyperintense signal, due to mural hematoma (asterisk), consistent with vessel dissection (b).

\section{Disclosure Statement}

The authors have no conflicts of interest to disclose.

Reference

Pelkonen O, Tikkakoski T, Pyhtinen J, Sotaniemi K: Cerebral CT and MRI findings in cervicocephalic artery dissection. Acta Radiol 2004;45:259-265.
MRI scans (2 days after the onset of left hemiplegia) showing increased signal on diffusion-weighted imaging (c), with decreased signal on ADC maps (d), indicating cytotoxic edema due to ischemic lesion in the right frontoparietal region. 